Editorial

\title{
Una perspectiva internacional sobre la formación en materia de seguridad y salud en el trabajo
}

An international perspective on occupational safety and health training

\section{Félix Martín Daza}

Centro Internacional de Formación de la Organización Internacional del Trabajo (OIT). Ginebra. Suiza.

\section{Correspondencia}

Félix Martín Daza

Centro Internacional de Formación de la Organización Internacional del Trabajo (OIT).

Ginebra. Suiza

Correo electrónico: F.Martin@itcilo.org

Resumen

El presente artículo tiene el propósito de explorar algunos de los retos más importantes de la formación en la seguridad y salud en el trabajo de los diferentes grupos interesados, así como para presentar los lineamientos de la Organización Internacional del Trabajo en esta materia.

Med Segur Trab (Internet) 2013; 59 (231) 171-175

Palabras clave: formación, OIT, seguridad, salud, trabajo.

\section{Abstract}

The present article has the propose of exploring some of the most important challenges of the training of different groups on occupational safety and health as well as to present the guidelines of the International Labour Organization in this subject.

Med Segur Trab (Internet) 2013; 59 (231) 171-175

Key words: Training, ILO, safety, health, work. 


\section{INTRODUCCIÓN}

Una adecuada educación y formación de los principales actores a nivel de empresa es una condición previa para el buen desempeño en materia de seguridad y salud en el trabajo (SST), y para la reducción progresiva de los accidentes de trabajo y enfermedades profesionales. Durante muchos años la Organización Internacional del Trabajo ha promovido, a través de las normas internacionales del trabajo, la necesidad de formación en materia de SST para aquellos con responsabilidades (gerentes, supervisores, trabajadores y sus representantes, inspectores, etc.). Aunque muchos países ya han incluido requisitos legales en esta materia, todavía hay muchos países que no tienen base legal para la formación en materia de SST. Muchas veces, cuando existe, no hay una oferta nacional adecuada (tanto en la cantidad y calidad) para satisfacer dichas necesidades de formación. Sin embargo, las experiencias de algunos países demuestran que, con diferentes enfoques y alternativas, se pueden observar mejoras notables en la capacidad de formación, en un período relativamente corto de tiempo. Las autoridades públicas y las partes interesadas deberían reflexionar sobre la mejor manera de construir un sistema que proporcione formación en SST para personas con responsabilidades en este tema, que sea a la vez eficaz y sostenible.

Este artículo tiene el propósito de explorar las necesidades de formación en SST y algunos de los retos más importantes para la formación de los diferentes grupos en SST, especialmente en los países en desarrollo.

\section{LAS NORMAS INTERNACIONALES DEL TRABAJO Y LA FORMACIÓN EN MATERIA DE SST}

Las normas internacionales del trabajo de la Organización Internacional del Trabajo (OIT) contienen numerosas referencias a la cuestión de la formación y la educación de SST. Por ejemplo, la formación se incluye como una de las principales áreas de acción debe ser contemplada en la Política Nacional de la SST (Convenio 155, artículo 5c) y una parte integral del Sistema Nacional de SST (Convenio 187, artículo 3c), que es la infraestructura necesaria para asegurar el buen desempeño en materia de SST a nivel nacional. En este sentido, los Estados Miembros deben "promover mecanismos para impartir educación y formación sobre seguridad y salud en el trabajo, en particular a los directores, los supervisores, los trabajadores y sus representantes, y a los funcionarios encargados de la seguridad y la salud" (como propuesto en la Recomendación 197, Artículo 5b).

Las normas internacionales del trabajo no son tan prescriptivas en la definición de la necesidad de la formación de especialistas en materia de SST, la consideran parte indispensable de los servicios de salud ocupacional en las empresas. Art. 36.2 de la Recomendación 171 sobre los servicios de salud en el trabajo dice que "deberían disponer de personal técnico en número suficiente, con formación especializada y experiencia en esferas tales como la medicina del trabajo, la higiene del trabajo, la ergonomía, los cuidados de enfermería del trabajo y otras cuestiones conexas".

Las directrices relativas a los sistemas de gestión de la SST (ILO OSH 2001), que es el documento de referencia de la OIT para la gestión de la SST en las empresas, indican en su artículo 3.4 las características que los programas de formación en materia de SST deben tienen que estar en conformidad con las siguientes directrices:

\section{(...) los programas de formación deberían:}

a) hacerse extensivos a todos los miembros de la organización, según sea pertinente;

b) ser impartidos por personas competentes;

c) ofrecer cuando proceda y de manera eficaz una formación inicial y cursos de actualización a intervalos adecuados;

d) comprender una evaluación por parte de los participantes de su grado de comprensión y retención de la capacitación;

e) ser revisados periódicamente, con la participación del comité de seguridad y salud, cuando exista, $y$ ser modificados según sea necesario para garantizar su pertinencia y eficacia, $y$

f) estar suficientemente documentados, y adecuarse al tamaño de la organización y a la naturaleza de sus actividades. 
Por lo tanto, las normas internacionales del trabajo y otros documentos pertinentes de la OIT proporcionan una referencia a la necesidad e importancia de la formación en SST, los grupos prioritarios que deben ser formados, así como los principios y directrices sobre cómo implementar la formación de manera eficiente.

\section{LA INTRODUCCIÓN DE LA SST EN LA FORMACIÓN}

Está bastante aceptado la necesidad y la importancia de la creación y la consolidación de una cultura de prevención en la sociedad, ya que es la base para apoyar la mejora progresiva de las condiciones de seguridad y salud. Para ello, es imprescindible la introducción de la SST en la educación básica para que los niños pudieran adquirir las actitudes preventivas. La OIT ya había abordado esta cuestión en 1981 en el convenio marco sobre seguridad y salud en el trabajo, el Convenio 155. La Convención establece en su artículo 14, que "deberán tomarse medidas a fin de promover, (...) la inclusión de las cuestiones de seguridad, bigiene y medio ambiente de trabajo en todos los niveles de enseñanza y de formación, incluidos los de la enseñanza superior técnica, médica y profesional, con objeto de satisfacer las necesidades de formación de todos los trabajadores."

La integración de la SST en el sistema educativo debería incluir tanto a cursos de educación general como de formación profesional (cuyos estudiantes serán los trabajadores del mañana) para promover la adquisición de los necesarios conocimientos, habilidades y actitudes preventivas que permitan la internalización de comportamientos seguros.

Aunque la presencia del tema de SST es común en los programas de estudios de formación profesional en muchos países, es bastante inusual su inclusión como tema transversal en todas las materias así como la formación práctica. La SST debería ser una parte esencial en el aprendizaje de los procesos de trabajo, en los que el aprendizaje de las conductas seguras y técnicas de trabajo debería estar estrechamente vinculado. Otro problema puede ser la falta de una gestión preventiva en sus propios centros de formación y la presencia de las tecnologías obsoletas e incluso peligrosas utilizadas en los talleres de aprendizaje práctico.

Algunas profesiones que requieren estudios universitarios implican la exposición a los riesgos de su actividad. Además, algunas de estas profesiones son clave en la prevención de riesgos para los trabajadores u otras personas, por ejemplo, arquitectos, ingenieros de producción o de organización, administradores de empresas, gerentes de recursos humanos, etc. Por tanto aunque la inclusión de la SST en algunos estudios universitarios sería también deseable y necesaria, es infrecuente en la mayoría de los países.

\section{LA FORMACIÓN DE DIFERENTES GRUPOS 1}

Los derechos de los trabajadores para acceder a la información y formación en SST está presente en prácticamente todas las normas de la OIT en la materia.

La formación a todos los niveles debe ser considerada como un medio de mejorar las condiciones de trabajo y medio ambiente. Los empleadores deben proporcionar la formación e instrucciones necesarias al personal, de acuerdo a la naturaleza de sus funciones y responsabilidades, el nivel técnico de su actividad y teniendo en cuenta las capacidades de las distintas categorías de trabajadores. Los programas de capacitación deben incluir los riesgos laborales existentes y potenciales del entorno de trabajo y centrarse en su prevención, control y protección.

Los trabajadores, en especial los recientemente incorporados, necesitan instrucciones sobre la seguridad y la salud de su trabajo y ser supervisados de cerca para asegurarse de que son plenamente conscientes de la naturaleza de los riesgos y cómo evitarlos. Además, los trabajadores y sus representantes deberían tener un número razonable de horas de trabajo remunerado para recibir su formación en materia de SST.

Además de los trabajadores, es muy importante la formación de otros grupos de personas con responsabilidades en materia de SST. Esto se aplica a los especialistas de SST, a los administradores de empresas, a los representantes de los trabajadores en SST, a los productores y fabricantes de maquinaria, etc. La formación de todos estos grupos es esencial para asegurar que la prevención de los riesgos es aplicada eficazmente y está verdaderamente integrada en las funciones de estos grupos. Sin embargo, no es frecuente

Una perspectiva internacional sobre la formación en materia de seguridad y salud en el trabajo 
que las legislaciones nacionales prevean la formación obligatoria para los empleadores, gerentes y ejecutivos en SST, aunque tengan las principales responsabilidades.

\section{LA FORMACIÓN DE ESPECIALISTAS EN SST ${ }^{2}$}

La formación de los especialistas que prestan apoyo para la gestión de la SST en la empresa suele estar regulada en los países desarrollados, no así en los países en desarrollo. Se estimula así la demanda de formación en este ámbito y por supuesto el desarrollo de la formación ofrecida por las instituciones.

La organización de la formación de especialistas en SST varía según los países. Esta heterogeneidad se puede encontrar tanto teniendo en cuenta:

- el nivel de formación (básico, intermedio o avanzado) requeridos por los especialistas;

- el tipo de institución que ofrece formación (título universitario o no, las instituciones acreditadas o no, etc.), y

- el nivel de especialización en el tema, por ejemplo, si la formación debe ser para la profesionales generalistas en SST o especializados en alguna disciplina en particular como higiene industrial, medicina del trabajo, seguridad en el trabajo, o ergonomía.

Las modalidades más comunes que pueden encontrarse en los diferentes países son:

- un título específico de formación profesional o de otro tipo de nivel medio, que no requiere una titulación universitaria previa;

- un título universitario en el nivel medio o superior tanto de carácter general en SST o varios más especializados en las diferentes disciplinas de SST;

- una formación de posgrado (que también puede ser de carácter general o especializado) proporcionada por una universidad.

La regulación incluye frecuentemente los contenidos de la formación, la acreditación de los centros autorizados, etc.

Es muy importante que las autoridades competentes en SST, prevean y planifiquen las necesidades del país en términos de la cantidad y calidad de los especialistas requeridos o el tipo y el nivel de conocimientos serían necesarias en un país en el mediano y largo plazo. Sin embargo, es común que no se hayan previsto estas necesidades y cuando se regula esta cuestión, sea una consecuencia y reacción a los problemas existentes. Sin embargo, a veces hay un requisito legislativo pidiendo las empresas para las funciones especializadas en materia de SST y, al mismo tiempo, la falta de capacidad en el país para capacitar en esa especialización. Por ejemplo, se requiere la vigilancia de la salud de los trabajadores, y al mismo tiempo, no existe una formación especializada en Medicina del Trabajo.

\section{LOS PROGRAMAS DE FORMACIÓN DE LOS ESPECIALISTAS EN SST}

Dado que la regulación en SST exige a las empresas la contratación de especialistas en SST, los poderes públicos deben garantizar de alguna manera una cierta garantía de calidad de estos especialistas. En general, esto requiere tomar varias medidas:

Regular el contenido de la formación, o mejor aún las competencias que estos especialistas deben obtener al final del proceso de formación. Básicamente, los especialistas deben adquirir las habilidades necesarias para realizar las actividades preventivas encomendadas a las empresas para las que van a trabajar. En general, la formación de un especialista en SST debería incluir la adquisición de un conjunto de diferentes tipos de competencias: técnicas, sociales, organizativas, de gestión, etc. En la mayoría de los países, se ha dado tradicionalmente un mayor énfasis en la formación de competencias de carácter técnico, confiando la adquisición de otras capacidades a la experiencia en el trabajo. El análisis de las funciones y tareas llevadas a cabo por especialistas en SST ha puesto de relieve la importancia y la necesidad de incluir el entrenamiento en estas habilidades.

Restringir el reconocimiento legal de la formación proporcionada por los centros acreditados por la autoridad competente, tras un análisis de sus programas, sus medios de formación y el equipo de formadores. 
Sin embargo, en algunos países, esta limitación podría tener efectos adversos sobre todo si se limita el número de especialistas que pueden ser capacitados con respecto a las necesidades del país.

Un elemento importante en la formación de especialistas es la adopción de un enfoque multidisciplinario. Ello significa que cualquier formación especializada que cubre todas las áreas o disciplinas preventivas (aunque se especialice en una de ellas). Esto podría ser a través de programas de capacitación que tienen un núcleo común y una parte especializada. La razón de este enfoque es proporcionar un lenguaje y conceptos comunes con el fin de preparar a especialistas para actuar tanto de forma individual como para colaborar en el trabajo de un equipo multidisciplinario.

Otro elemento importante se refiere a la necesidad de una oferta de formación continua y sistemática de los especialistas en SST (y para otros grupos como, por ejemplo, los inspectores de trabajo). Es necesario pues la presencia en cada país de uno o varios centros, instituciones u organizaciones que ofrezcan cursos de formación de corta duración para actualizar conocimientos sobre temas específicos de los especialistas en SST como, por ejemplo, nuevos peligros y medidas de prevención y protección; nueva legislación, políticas, procedimientos y protocolos; buenas prácticas nacionales e internacionales, nuevas tecnologías, procesos y formas de organización del trabajo, etc.

Algunos foros solicitan el desarrollo de un modelo de plan de estudios internacional para la formación de especialistas en SST que pudiera ofrecer un sistema de reconocimiento de estos estudios entre los diferentes países. Esta propuesta está motivada por la posibilidad de que estos especialistas puedan acceder a puestos de trabajo en diferentes países, o la posibilidad de estudios especializados en materia de SST en diversos países. Podría ser una perspectiva interesante sobre todo para las empresas multinacionales y también para los países en desarrollo (considerada la falta de recursos de formación a nivel local en muchos de ellos).

Por otro lado, puede ser necesario disponer de una acomodación nacional del plan de estudios para adaptarse a las características de cada país, teniendo en cuenta el número de empresas, su tamaño o su distribución por sectores, la legislación especial, o su nivel de desarrollo, etc. Por ejemplo, en algunos países en su actual nivel de desarrollo, podría ser más necesario el desarrollo de la capacidad de formación de tener un gran número de especialistas en SST en un nivel básico (que podrían estar presentes en un gran número de empresas), en lugar de dar prioridad a la formación avanzada que ofrecen las universidades (que restringen el número de especialistas en SST).

También es muy importante tener en cuenta los requisitos establecidos en las legislaciones nacionales en materia de SST tanto en la necesidad de que las empresas tengan recursos de prevención internos y externos, así como el papel que la ley les confiere.

Estas iniciativas pueden tener un valor significativo para las áreas internacionales con un alto grado de homogeneidad, como la Unión Europea. La realidad es que, incluso con la legislación de SST del origen común y un alto grado de intercambio de conocimientos entre los países, existe una gran diversidad en la forma en que la formación de especialistas en SST se organiza.

\section{REFERENCIAS BIBLIOGRÁFICAS}

1. Ali, Benjamin, Principios fundamentales de la seguridad y salud en el trabajo. Oficina Internacional del Trabajo, 2001

2. Castellá, José Luis, Guía de introducción a los Sistemas Nacionales de Seguridad y Salud en el Trabajo. Documento de Trabajo de la OIT, 2002. 